\title{
Cavity QED with chip-based toroidal microresonators
}

B. Dayan, T. Aoki, E. Wilcut, S. Kelber, W. P. Bowen, et al.

B. Dayan, T. Aoki, E. Wilcut, S. Kelber, W. P. Bowen, A. S. Parkins, J. R. Petta, T. J. Kippenberg, E. Ostby, K. J. Vahala, H. J. Kimble, "Cavity QED with chip-based toroidal microresonators," Proc. SPIE 6710, Quantum Communications and Quantum Imaging V, 67100H (3 October 2007); doi: $10.1117 / 12.734875$

EPIE Event: Optical Engineering + Applications, 2007, San Diego, California, United States 


\title{
Cavity QED with chip-based toroidal microresonators
}

\author{
B. Dayan ${ }^{\mathrm{a}}$, T. Aoki ${ }^{\mathrm{a}}$, E. Wilcut ${ }^{\mathrm{a}}$, S. Kelber ${ }^{\mathrm{a}}$, W. P. Bowen ${ }^{\text {1a }}$, A. S. Parkins ${ }^{2 \mathrm{a}}$, J. R. Petta ${ }^{3 \mathrm{a}}$, T. J. \\ Kippenberg ${ }^{4 b}$, E. Ostby ${ }^{b}$, K. J. Vahala ${ }^{\text {b }}$, H. J. Kimble ${ }^{a}$ \\ ${ }^{a}$ Norman Bridge Laboratory of Physics 12-33, California Institute of Technology, Pasadena, \\ California 91125, USA. \\ ${ }^{\mathrm{b}} \mathrm{T}$. J. Watson Laboratory of Applied Physics, California Institute of Technology, Pasadena, \\ California 91125, USA.
}

\begin{abstract}
We report the demonstration of strong coupling between single Cesium atoms and a high-Q chip-based microresonator. Our toroidal microresonators are compact, Si chip-based whispering gallery mode resonators that confine light to small volumes with extremely low losses, and are manufactured in large numbers by standard lithographic techniques. Combined with the capability to couple efficiently light to and from these microresonators by a tapered optical fiber, toroidal microresonators offer a promising avenue towards scalable quantum networks. Experimentally, laser cooled Cs atoms are dropped onto a toroidal microresonator while a probe beam is critically coupled to the cavity mode. When an atom interacts with the cavity, it modifies the resonance spectrum of the cavity, leading to rejection of some of the probe light from the cavity, and thus to an increase in the output power. By observing such transit events while systematically detuning the cavity from the atomic resonance, we determine the maximal accessible single-photon Rabi frequency of $\Omega_{0} / 2 \pi \approx(100 \pm 24) \mathrm{MHz}$. This value puts our system in the regime of strong coupling, being significantly larger than the dissipation rates in our system.
\end{abstract}

Keywords: Cavity Quantum Electrodynamics Microresonators Quantum Optics Cold Atoms

\section{INTRODUCTION}

Over the past decade, strong interactions of light and matter at the single-photon level have enabled a wide set of scientific advances in Quantum Optics and Quantum Information Science. Experiments with single, localized atoms have been at the forefront of these advances by utilizing optical resonators formed by two spherical mirrors in a FabryPerot configuration ${ }^{1}$. Due to the extreme technical challenges involved in further improvement of these coatings and in scaling to large numbers of such resonators, there has been increased interest in the development of alternative microcavity systems. For example, the realization of large-scale quantum networks requires the capability to interconnect many "quantum nodes," each of which could consist of a microresonator containing a set of trapped atoms. The "quantum channels" to connect these nodes would be optical fiber, with strong interactions in cavity QED providing an efficient interface between light and matter ${ }^{2,3}$.

Our efforts have centered on the whispering-gallery modes (WGMs) of fused silica microtoroidal resonators ${ }^{4-7}$, which have demonstrated quality factors $\mathrm{Q} \approx 4 \times 10^{8}$. These resonators are fabricated on silicon wafers in large numbers by standard lithographic techniques followed by a laser-reflow process. Combined with efficient coupling of light to and from such cavities via a tapered optical fiber $^{5}$, toroidal microcavities offer promising capabilities for new nonlinear interactions of single atoms and photons across distributed networks ${ }^{8}$.

\footnotetext{
${ }^{1}$ Present address: Physics Department, University of Otago, Dunedin 9016, New Zealand.

${ }^{2}$ Present address: Department of Physics, University of Auckland, Auckland 1142, New Zealand.

${ }^{3}$ Present address: Department of Physics, Princeton University, Princeton, New Jersey 08544, USA.

${ }^{4}$ Present address: Max Planck Institute of Quantum Optics, Garching 85748, Germany.
} 


\section{THE EXPERIMENT}

In Ref. [9], we report a new paradigm for cavity QED with single atoms and photons. Specifically, we achieve strong coupling between single Cesium atoms and the fields of a microtoroidal cavity fabricated from $\mathrm{SiO}_{2}$. As illustrated in Figure 1, laser cooled atoms are dropped from above the microtoroid, with some small number of atoms transiting through the external evanescent field of the resonator. The interaction of one atom with the evanescent field destroys the condition of critical coupling, leading to an increase in the forward propagating power $P_{F}$ in the fiber taper. By recording the dependence of $P_{F}$ on the frequency detuning $\Delta_{A C}$ between the atom and cavity resonances for individual atom transits, we infer the maximum accessible coupling rate $g^{m}{ }_{0} / 2 \pi=50 \pm 12 \mathrm{MHz}$ by way of comparisons to a new theoretical model. Our system thereby achieves the conditions for strong coupling, namely $g^{m}{ }_{0}>\left(\gamma, \kappa, \Delta t^{-1}\right)$, where $g^{m}{ }_{0}$ is half the single-photon Rabi frequency, the rate of atomic spontaneous decay $\gamma / 2 \pi=2.6 \mathrm{MHz}$, the cavity field decay rate $\kappa / 2 \pi=18 \pm 3 \mathrm{MHz}$, and $\Delta t \approx 2 \mu$ s is the average transit duration through the evanescent field of the toroidal resonator.

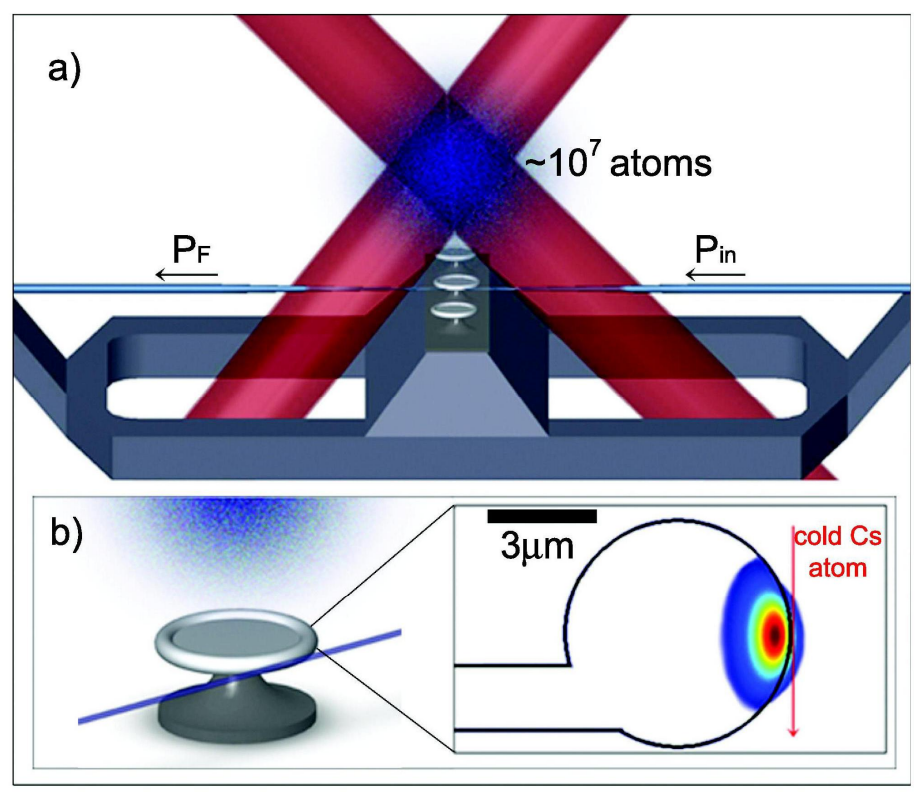

Fig. 1. A simple diagram of the experiment (Ref [9]). A cloud of cold Cesium atoms and the associated trapping lasers above an array of microtoroidal resonators. Light from the probe beam $P_{\text {in }}$ is coupled into one of these resonators resonator by way of the fiber taper, with the forward propagating output $P_{\mathrm{F}}$ coupled back into the taper from the resonator. (b) Illustration of an $\mathrm{SiO} 2$ microtoroidal resonator, fiber taper, and atom cloud above. The calculated field distribution for the lowest order resonator mode is shown by the color contour plot on the right. Cold Cesium atoms fall through the external evanescent field of this mode and are thereby strongly coupled to the resonator's field.

Our measurements are the first to demonstrate strong coupling for single atoms interacting with an optical resonator other than a conventional Fabry-Perot cavity. We estimate that the efficiency for coupling single photons into and out of our microtoroidal resonators could approach $\varepsilon \sim 0.99$, while still remaining firmly in the regime of strong coupling ${ }^{5}$. Such high efficiency is critical for the realization of scalable quantum networks and photonic quantum computation. Indeed, of diverse possibilities for the distribution and processing of quantum information with optical cavities, the system of single atoms coupled to microtoroidal resonators arguably provides one of the most promising avenues. 
Acknowledgements We thank M. Eichenfield, K. W. Goh and S. M. Spillane for their contributions to the early stages of this experiment, and T. Carmon, A. Gross and S. Walavalker for their contributions to the current realization.

The work of H.J.K. is supported by the National Science Foundation, the Disruptive Technology Office of the Department of National Intelligence, and Caltech. The work of K.J.V. is supported by DARPA, the Caltech Lee Center and the National Science Foundation. B.D., W.P.B. and T.J.K. acknowledge support as Fellows of the Center for the Physics of Information at Caltech. A.S.P. acknowledges support from the Marsden Fund of the Royal Society of New Zealand. The Work of TA is supported by Japan Science and Technology Agency (JST).

\section{REFERENCES}

1. R. Miller et al., "Trapped atoms in cavity QED: coupling quantized light and matter", J. Phys. B At. Mol. Opt. Phys. 38, S551-S565 (2005).

2. J. I. Cirac et al., "Quantum state transfer and entanglement distribution among distant nodes in a quantum network", Phys. Rev. Lett. 78, 3221-3224 (1997).

3. H. -J. Briegel et al., in The Physics of Quantum Information, edited by D. Bouwmeester, A. Ekert, and A. Zeilinger, 192-197 (Springer, Berlin, 2000).

4. D. K. Armani et al., "Ultra-high- $Q$ toroid microcavity on a chip", Nature 421, 925-928 (2003).

5. S. M. Spillane et al., "Ideality in a fiber-taper-coupled microresonator system for application to cavity quantum electrodynamics", Phys. Rev. Lett. 91, 043902 (2003).

6. T. J. Kippenberg, S. M. Spillane and K. J. Vahala, "Demonstration of ultra-high- $Q$ small mode volume toroid microcavities on a chip", Appl. Phys. Lett. 85, 6113-6115 (2004).

7. K. J. Vahala, "Optical microcavities", Nature 424, 839-846 (2003) and references therein.

8. S. M. Spillane et al., "Ultrahigh-Q toroidal microresonators for cavity quantum electrodynamics", Phys. Rev. A. 71, 013817 (2005).

9. T. Aoki, et al., "Observation of strong coupling between one atom and a monolithic microresonator", Nature 443, 671-674 (2006). 